\title{
Montaje para la determinación in situ por espectrometría gamma de descargas líquidas de ${ }^{131}$ I en servicios de terapia
}

\section{A setup for in situ measurements of liquid discharges of ${ }^{131}$ l by gamma spectrometry in radiotherapy services}

\author{
F. Mosos, A. Abadía, J.W. Gómez y P. Casallas \\ ${ }^{1}$ Dirección de Asuntos Nucleares, Servicio Geológico Colombiano, Carrera 50 No. 26-20, Bogotá, D.C. Colombia \\ Autor para correspondencia:fmosos@sgc.gov.co
}

\begin{abstract}
Resumen
En este trabajo se presenta una alternativa para la determinación de la actividad descargada de ${ }^{131} \mathrm{I}$ en los vertimientos de las instalaciones de medicina nuclear que realizan terapia. Se desarrolla un montaje para la determinación in situ de descargas líquidas de ${ }^{131}$ I mediante la técnica de espectrometría gamma de alta resolución utilizando un detector de germanio hiperpuro. El montaje construido simuló las condiciones reales de descarga en una instalación, con el propósito de poner a punto el sistema de medida empleado, determinando los parámetros de eficiencia, incertidumbre y límite de detección.
\end{abstract}

Palabras clave: ${ }^{131}$ I, Descargas radiactivas, Detector de GeHp, Yodo, Espectrometría Gamma in situ

\begin{abstract}
This paper presents an alternative for determining the activity discharged ${ }^{131} \mathrm{I}$ in the dumping of nuclear medicine facilities that perform therapy, a mount for the in situ determination of liquid discharges of ${ }^{131} \mathrm{I}$ by spectrometry technique developed gamma high resolution using hyperpure germanium detector. The assembly constructed simulated actual discharge conditions in an installation, in order to fine-tune the measurement system used, determining the parameters of efficiency, uncertainty and detection limit.
\end{abstract}

Key words: ${ }^{131} \mathrm{I}$, Radioactive discharges, HpGe Detector, Iodine, in situ gamma-ray spectrometry

\section{INTRODUCCIÓN}

El empleo de compuestos radiactivos en la medicina es de amplio uso para el diagnóstico y tratamiento de muchas enfermedades. En Colombia, la medicina nuclear se inició en 1955 con la aplicación de yodo-131 ( $\left.{ }^{131} \mathrm{I}\right)$ [1], el cual es ampliamente empleado a nivel mundial [2-3], donde se administran actividades terapéuticas que van desde 800 a $6000 \mathrm{MBq}$, dependiendo de la enfermedad a tratar [4]. Estos valores son muy superiores a los usados en diagnóstico, y por tanto representan un riesgo radiológico significativo.
De la actividad total de ${ }^{131} \mathrm{I}$ administrada a un paciente en un tratamiento, entre el 70 y el $90 \%$ se elimina por la orina en las primeras 48 horas. En la actualidad las instalaciones que usan este radionúclido en pacientes, con fines de terapia, mantienen a las personas hospitalizadas en cuartos que han sido diseñados para dirigir la orina a sistemas de manejo de vertimientos [5-6], donde se deben retener los líquidos hasta que alcancen niveles de radiactividad por debajo de los límites autorizados [7]. 
Las instalaciones generadoras de vertimientos deben medir la magnitud de las descargas radiactivas de ${ }^{131}$ I con el fin de verificar que no se superen los límites establecidos en la normatividad de cada país. De acuerdo a la Resolución 41178 del Ministerio de Minas y Energía de Colombia [7-8], la cantidad límite de vertido líquido a masas de agua para ${ }^{131} \mathrm{I}$ es de $1 \times 10^{7} \mathrm{~Bq} /$ año. La medición de las descargas radiactivas de ${ }^{131} \mathrm{I}$ requiere equipos con la capacidad de medir radiación gamma en bajas concentraciones (del orden de $1 \times 10^{2} \mathrm{~Bq} / \mathrm{l}$ ), por esta razón usualmente se emplean metodologías de muestreo y medición en laboratorio [9-10]. Debido a los requisitos regulatorios relacionados con estas descargas, cada instalación debe contar con un sistema para el manejo adecuado de los vertimientos, que además de representar un riesgo radiológico, implica también un riesgo biológico.

La alternativa de determinar la concentración radiactiva de ${ }^{131} \mathrm{I}$ in situ ofrece ventajas técnicas y de seguridad sobre una metodología que implique el muestreo y posterior análisis en laboratorio [11], entre las que sobresalen:

- No hay contacto físico de los vertimientos con el equipo de medida ni es necesaria la manipulación de muestras radiactivas, que además presentan riesgos biológicos.

- Los resultados se obtienen en forma inmediata en el lugar de medición, obviando los tiempos generalmente largos de las mediciones de laboratorio.

- No se generan desechos radiactivos adicionales a nivel de laboratorio por cuanto no es necesaria la manipulación de muestras.

Con base en lo expuesto anteriormente, el propósito de este trabajo consiste en diseñar un montaje experimental para la determinación in situ de descargas líquidas de ${ }^{131} \mathrm{I}$, con miras a su posterior implementación dentro de un protocolo en servicios de terapia. En el contexto actual es relevante contar con una alternativa técnica de evaluación de vertimientos que sea de fácil implementación manteniendo criterios de trazabilidad metrológica y la capacidad de dar resultados con incertidumbre y límites de detección suficientemente bajos para ser aplicable en la práctica.

\section{METODOLOGÍA}

\subsection{Equipo de espectrometría gamma}

El trabajo experimental se desarrolló en la Instalación Centralizada para la Gestión de Desechos Radiactivos perteneciente al Servicio Geológico Colombiano. El equipo usado en la investigación es un sistema portátil de espectrometría gamma de alta resolución con detector de germanio hiperpuro (GeHp), el cual tiene una eficiencia relativa del $10 \%$ en la energía de 1,33 MeV. En la Tabla 1 se presentan las principales especificaciones del equipo:

Tabla 1. Especificaciones del sistema de espectrometría portátil [12]

\begin{tabular}{l|l}
\hline \multicolumn{1}{c|}{ Especificación } & \multicolumn{1}{c}{ Valor } \\
\hline Marca & Canberra \\
\hline Modelo del detector & GL0515 \\
\hline Multicanal & MCA-527 \\
\hline Dimensiones del detector & $\begin{array}{l}\text { Área: } 500 \mathrm{~mm}^{2} \\
\text { Espesor: } 15 \mathrm{~mm}\end{array}$ \\
\hline Autonomía del criostato & $22 \mathrm{~h}$ \\
Intervalo energético & $>5 \mathrm{keV}$ \\
Resolución para $122 \mathrm{keV}$ & $0,55 \mathrm{keV}$ \\
\hline
\end{tabular}

El ${ }^{131} \mathrm{I}$ es un radionúclido con periodo de semidesintegración de 8,02 días [13] es emisor beta y gamma. Las energías de emisión gamma características se muestran en la Tabla 2, el fotópico de $364 \mathrm{keV}$ se seleccionó para realizar la cuantificación de la actividad debido a su alto rendimiento gamma.

Tabla 2. Fotones gamma característicos del 131I [13]

\begin{tabular}{c|c}
\hline Energía $[\mathrm{keV}]$ & Rendimiento gamma \\
\hline 80,1850 & 0,02607 \\
\hline 284,305 & 0,0606 \\
\hline 364,489 & 0,812 \\
\hline 636,989 & 0,0726 \\
\hline 722,911 & 0,0196 \\
\hline
\end{tabular}




\subsection{Marco teórico para la medición}

Para modelar las descargas líquidas de ${ }^{131} \mathrm{I}$ y desarrollar la metodología de medición, se parte de la definición del punto de descarga como una caja que recibe los vertimientos de la instalación y entrega los mismos a la red de alcantarillado. La caja es modelada como un paralelepípedo que mantiene un nivel de líquido constante o bien, el nivel varía en función de la tasa de descarga. Dicha caja de inspección normalmente se construye en concreto con un área transversal que se aproxima a un cuadrado de $60 \mathrm{~cm}$ de lado [14], que en condiciones normales se encuentra cerrada con una tapa que usualmente se construye de concreto.

En este montaje, la medición se realiza con la caja de inspección destapada. El detector se ubica a una distancia vertical determinada sobre la línea central simétrica, tal como se presenta en la Figura 1. Con el fin de reproducir las condiciones de medición, para posicionar el detector en una geometría reproducible, se utilizó una lámina de acrílico sobre la caja de inspección que cuenta con un agujero en la parte central lo suficientemente amplio para evitar interacción del campo de radiación generado desde la matriz hacia el detector.

Figura 1. Esquema del punto de descarga (caja de inspección)

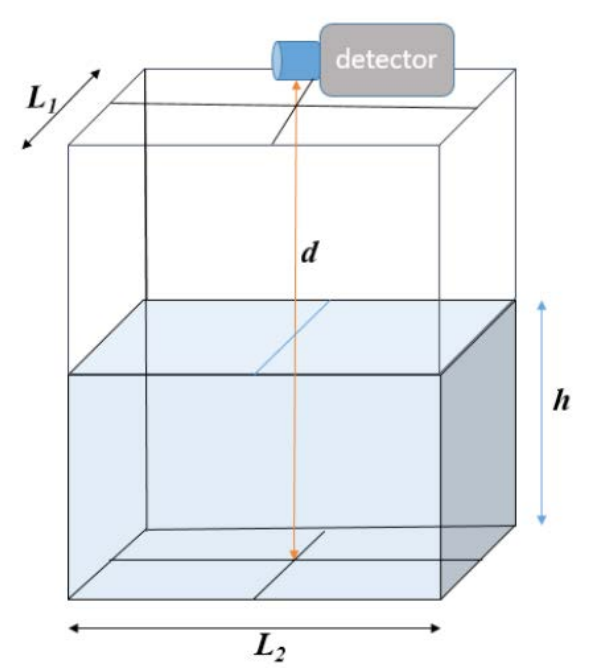

Donde:

$d$ : Distancia desde la base de la caja y la superficie lateral del detector $[\mathrm{cm}]$

$h$ : Altura de la columna de líquido [cm]

$L_{1}$ : Ancho interno de la caja $[\mathrm{cm}]$
$L_{2}:$ Largo interno de la caja $[\mathrm{cm}]$

En condiciones de estado estacionario, la actividad total que se descarga está dada por la Ec. 1:

$$
A_{T}=\int_{0}^{t} Q \times C d t
$$

Donde:

$A_{T} \quad$ Actividad total de ${ }^{131} \mathrm{I}$ descargada en el intervalo de tiempo $t[\mathrm{~Bq}]$

Q: Caudal de vertimiento $\left[1 \mathrm{~s}^{-1}\right]$

C: Concentración radiactiva de ${ }^{131} \mathrm{I}\left[\mathrm{Bq}^{-1}\right]$

Suponiendo una distribución homogénea del radionúclido en el líquido, lo cual es aplicable para ${ }^{131} \mathrm{I}$, la concentración radiactiva se puede determinar mediante espectrometría gamma a partir de la siguiente ecuación:

$$
C=\frac{R_{s}}{\varepsilon \gamma C_{f} V}
$$

Donde:

C: Concentración radiactiva $\left[\mathrm{Bq} \mathrm{l}^{-1}\right]$

$\varepsilon$ : Eficiencia de detección en el pico de absorción total para la energía de $364 \mathrm{keV}$

$V: \quad$ Volumen del líquido en la caja [1] $=h L_{1} L_{2} / 1000$

$C_{f}:$ Factor de corrección por decaimiento durante la medición

$R_{s}$ : Tasa de conteo neta en el fotópico característico $\left[\mathrm{s}^{-1}\right]$

$\gamma$ : Rendimiento gamma en la energía característica

Si la medición espectrométrica se realiza en un tiempo representativo, es posible tomar el caudal como el promedio constante que sale de la integral de la Ec. 1. De la misma manera, suponiendo que es posible estimar el volumen promedio del líquido en la caja durante el tiempo de medición, la actividad total descargada durante la medición se puede calcular a partir de la ecuación: 


$$
A_{T}=\frac{Q}{\varepsilon \gamma C_{f} \bar{V}} \int_{0}^{t} R_{s} d t
$$

Donde $\overline{\mathrm{V}}$ es el volumen promedio del líquido en la caja durante la medición. La integral corresponde al área bajo la curva gaussiana en el fotópico de $364 \mathrm{keV}$ del espectro obtenido, es decir, las cuentas netas en el fotópico $\left(N_{s}\right)$. La Ec. 3 se simplifica a la forma:

$$
A_{T}=\frac{Q N_{S}}{\varepsilon \gamma C_{f} \bar{V}}
$$

\subsection{Diseño y montaje experimental}

El objetivo del experimento es determinar la eficiencia de detección para la geometría del montaje (caja de inspección y sistema de medición, Figura 1) para diferentes valores de $d$ y $h$. A partir de los resultados de la eficiencia y los valores de $d$ y $h$ se deduce una expresión de la eficiencia en función de $d$ y $h$ que puede ser empleada para hacer determinaciones en las cajas de paso de las instalaciones que realizan terapias con ${ }^{131} \mathrm{I}$.

Para la realización de la investigación se procedió a construir un montaje con las dimensiones típicas de las cajas de inspección utilizadas en los centros médicos de la ciudad de Bogotá, cuya área transversal fue de $60 \mathrm{~cm} 60 \mathrm{~cm}$. Se utilizó material acrílico de $5 \mathrm{~mm}$ de espesor en las paredes y en la base y se rodeó completamente de bloques de concreto de $20 \mathrm{~cm}$ de espesor (ver Figura 2). El montaje se construyó de tal manera que se facilite el llenado $\mathrm{y}$ vaciado con soluciones acuosas de actividad conocida de ${ }^{131} \mathrm{I}$; además dadas las características del acrílico, fue factible medir las variables $h$ y $d$ con exactitud. Se dispuso de una lámina de acrílico superior que permitió la ubicación del detector a las distancias $d$ deseadas (Figura 2). Con el fin de no disminuir la eficiencia de detección, se perforó la parte central de la lámina superior, haciendo insignificante la atenuación de fotones entre el agua $y$ el detector.
Aunque la situación real para las mediciones en campo está prevista para realizarse en cajas de inspección cuyo material es concreto, se considera que en el montaje propuesto no es significativo el error asociado a la diferencia en la retrodispersión que existe entre el acrílico y el concreto debido al bajo espesor del acrílico y al bajo rendimiento gamma de las energías características de emisión del ${ }^{131} \mathrm{I}$ superiores a $364 \mathrm{keV}$ (ver Tabla 2).

Figura 2. Montaje experimental de una caja de inspección para la determinación de descargas in situ de ${ }^{131} \mathbf{I}$

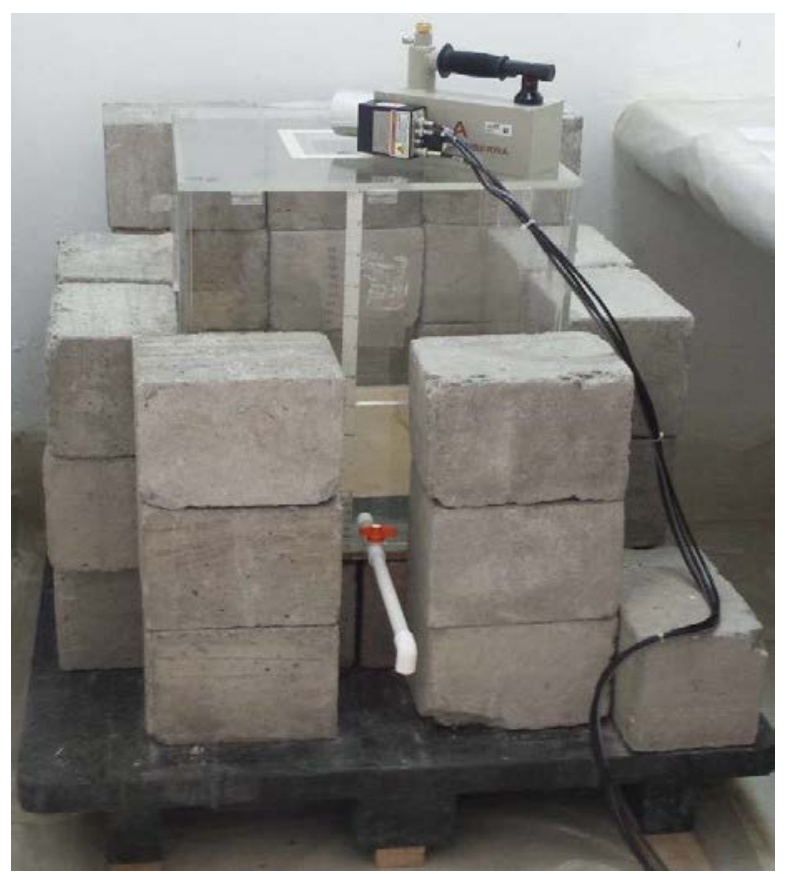

El experimento consistió en la preparación de una solución acuosa de ${ }^{131} \mathrm{I}$ de concentración de actividad conocida y la adquisición de espectros gamma para valores de $d$ y $h$ cuidadosamente elegidos con la finalidad de determinar la eficiencia experimental para cada caso y así estimar la eficiencia en el punto de descarga de la instalación que se debe usar en la Ec. 4. Los valores de $d$ usados fueron $62,0 \mathrm{~cm}, 69,7 \mathrm{~cm}$ y 77,4 $\mathrm{cm}$ y para cada uno de estos se usaron valores $h$ de 1,7 $\mathrm{cm}, 3,1 \mathrm{~cm}, 4,6 \mathrm{~cm}, 6,0 \mathrm{~cm}, 7,5 \mathrm{~cm}, 8,9 \mathrm{~cm}$ y $10,4 \mathrm{~cm}$. La elección de los valores de $d$ se basó en la necesidad de representar las condiciones previstas para la medición in situ; es decir, en la práctica y de acuerdo a lo exigido en la norma de cajas de inspección [14], estas deberán 
tener una altura de $60 \mathrm{~cm}$, por lo tanto, el detector se ubicó a una altura un poco superior. En cuanto a los valores de $h$ se tomaron valores de 0 a $10 \mathrm{~cm}$ ya que la norma mencionada anteriormente indica un rebose máximo de $10 \mathrm{~cm}$ para estas cajas.

\subsection{Calibración del sistema}

La calibración del sistema se realizó usando fuentes radiactivas con geometría puntual y actividad certificada por Czech Metrological Institute, para los radionúclidos ${ }^{133} \mathrm{Ba},{ }^{137} \mathrm{Cs}$ y ${ }^{60} \mathrm{Co}$. La eficiencia de detección se obtuvo como función de la energía a diferentes distancias fuente-detector. La curva de eficiencia para una geometría puntual permitió determinar la actividad de una fuente de ${ }^{131} \mathrm{I}$ de uso médico en forma de cápsula, resultando una actividad de (175 \pm 5$)$ MBq. Teniendo en cuenta las dimensiones de la cápsula $(5 \mathrm{~mm})$ y la distancia fuente detector usada en la estimación de la actividad (1856 mm) se consideró que se cumplían las condiciones para realizar esta calibración gruesa.

Posteriormente se diluyó a volumen conocido, se realizó el fraccionamiento $1 / 10$ y se tomó dicha fracción de esta solución, la cual se llevó hasta 35 litros de agua. La solución resultante fue la empleada para realizar las posteriores mediciones con el fin de determinar la eficiencia del equipo en el pico de absorción total para la energía de $364 \mathrm{keV}$, para el montaje en la caja de inspección.

\subsection{Estimación de incertidumbre}

La incertidumbre en la determinación de la eficiencia del equipo en el pico de absorción total se determinó como la incertidumbre combinada obtenida a partir de los aportes de incertidumbre de los parámetros relevantes para el experimento, que corresponden a las magnitudes de la Ec. 2 y a $d$ y $h$ que deben ser medidos para cada experimento. Para realizar lo anterior se usó la siguiente expresión:

$u_{\varepsilon}=\varepsilon \cdot \sqrt{\left(\frac{u_{A}}{A}\right)^{2}+\left(\frac{u_{m}}{m}\right)^{2}+\left(\frac{u_{\gamma}}{\gamma}\right)^{2}+\left(\frac{u_{c_{f}}}{c_{f}}\right)^{2}+\left(\frac{u_{d}}{d}\right)^{2}+\left(\frac{u_{h}}{h}\right)^{2}+\frac{\left(u^{2} R_{s+b}+u^{2} R_{b}\right)}{\left(R_{s+b}-R_{b}\right)^{2}}}$

Donde:

$u_{i}: \quad$ Incertidumbre de la magnitud $i$
A: Actividad de ${ }^{131}$ I del líquido en la caja $[\mathrm{Bq}]$

$m$ : Masa del líquido en la caja $[\mathrm{kg}]$

$R_{s+b}:$ Tasa de conteo total en el fotópico característico $\left[\mathrm{s}^{-1}\right]$

$R_{b}$ : Tasa de conteo de fondo en el fotópico característico $\left[\mathrm{s}^{-1}\right]$

\section{RESULTADOS Y DISCUSIÓN}

\subsection{Eficiencia}

En la Tabla 3 se muestran los resultados de los valores experimentales de eficiencia en el pico de absorción junto a la incertidumbre combinada $u$ obtenida para cada medida como función de las dos variables geométricas principales $h$ y $d$. En la Figura 3 se muestra la variación gráfica de $\varepsilon$ en función de $h$ para cada distancia $d$ medida.

Tabla 3. Resultados de mediciones de $\varepsilon$ para diferentes valores de $d \mathrm{y} h$

\begin{tabular}{|c|c|c|c|c|c|c|}
\hline \multirow{3}{*}{$\begin{array}{c}\text { altura } \\
\text { columna } \\
\text { líquido } h \\
(\mathrm{~cm})\end{array}$} & \multicolumn{6}{|c|}{ valores de $d$ empleados $(\mathrm{cm})$} \\
\hline & \multicolumn{2}{|c|}{62,0} & \multicolumn{2}{|c|}{69,7} & \multicolumn{2}{|c|}{77,4} \\
\hline & $\varepsilon \times 10^{7}$ & $\mathbf{u} \times 10^{9}$ & $\varepsilon \times 10^{7}$ & u $\times 10^{9}$ & $\varepsilon \times 10^{7}$ & u x $10^{9}$ \\
\hline 1,7 & 16 & $4 \mathrm{x}$ & 13 & 37 & 11 & 31 \\
\hline 3,1 & 7,2 & 21 & 5,9 & 17 & 4,9 & 14 \\
\hline 4,6 & 4,6 & 13 & 3,7 & 11 & 3,0 & 8,8 \\
\hline 6,0 & 3,3 & 9,4 & 2,7 & 7,7 & 2,2 & 6,3 \\
\hline 7,5 & 2,5 & 7,1 & 2,0 & 5,8 & 1,7 & 4,8 \\
\hline 8,9 & 2,0 & 5,8 & 1,6 & 4,7 & 1,3 & 3,8 \\
\hline 10,4 & 1,6 & 4,9 & 1,3 & 3,9 & 1,1 & 3,3 \\
\hline
\end{tabular}

Se observa que la eficiencia es mayor conforme se presentan menores valores de $h$. Este comportamiento es congruente con el hecho de que a mayor altura de la columna de líquido, el efecto de auto-atenuación aumenta. Se observa que para un mismo valor de $h$, la eficiencia disminuye con el aumento de la distancia $d$. Este comportamiento es más visible a menores valores 
de $h$ donde la variación de la eficiencia con el cambio de $d$ resulta de mayor magnitud. A partir de los resultados experimentales de la Tabla 3, se puede predecir el valor de eficiencia como función de $h$ y de $d$ para cualquier pareja de valores dentro del intervalo experimental estudiado, mediante la expresión:

$$
\varepsilon=a(d) \times h^{b(d)}
$$

Donde $a(d)$ se puede expresar a partir de una ecuación polinómica de segundo orden de la forma:

$$
a=a_{1} \times d^{2}+a_{2} \times d+a_{3}
$$
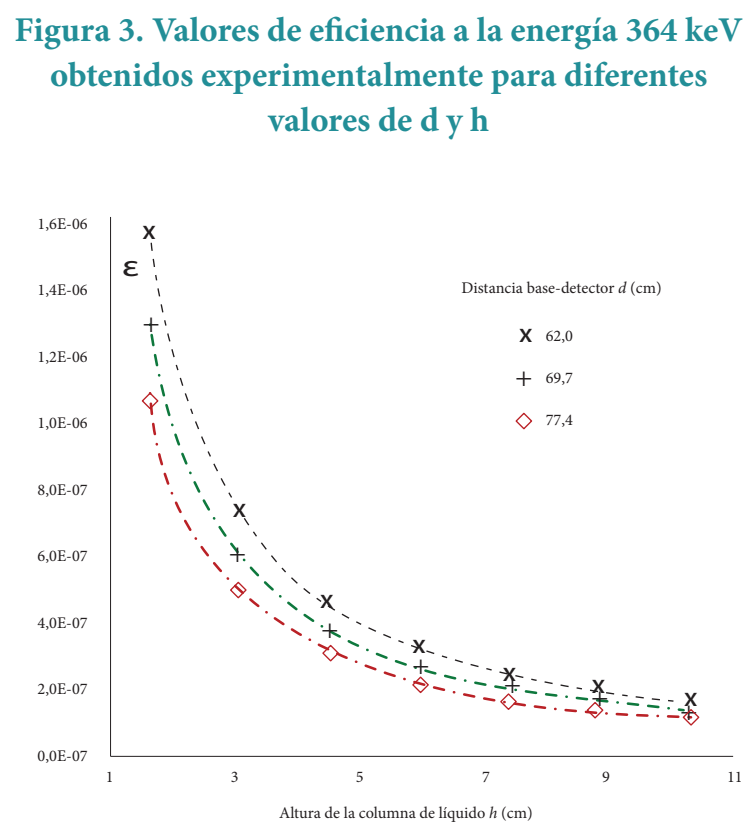

Los valores de $a_{1}, a_{2}$ y $a_{3}$, son constantes que se obtuvieron por regresión polinómica y son respectivamente: 7,3621 x $10^{-10},-1,6134 \times 10^{-7}$ y $-1,0066 \times 10^{-5}$. Por otro lado, $b(d)$ se determina por interpolación lineal de los valores de $b$ experimentales. A continuación, se presentan los resultados de los coeficientes $a$ y $b$ para los tres valores experimentales de $d$.
Tabla 4. Coeficientes a y b para la curva de ajuste de eficiencia

\begin{tabular}{c|c|c|c}
\hline $\mathbf{d}[\mathbf{c m}]$ & $\mathbf{6 2 , 0}$ & $\mathbf{6 9 , 7}$ & $\mathbf{7 7 , 4}$ \\
\hline$a$ & $2,8961 \times 10^{-06}$ & $2,3998 \times 10^{-06}$ & $1,9908 \times 10^{-06}$ \\
\hline$b$ & $-1,2255$ & $-1,2369$ & $-1,2386$ \\
\hline
\end{tabular}

\subsection{Capacidades para la determinación de ${ }^{131} \mathrm{I}$}

Con los resultados obtenidos es posible realizar mediciones caracterizando geométricamente el sistema con dos variables relevantes como lo son la altura de la columna de líquido y la diferente profundidad que pueda presentar la caja de inspección. Sin embargo, existen aún varios aspectos nombrados más adelante y que se deben estudiar y conocer más a fondo con el fin de continuar con la implementación del protocolo para medición in situ. Valores de eficiencia del orden de $10^{-6}$ como los obtenidos (ver Tabla 4) deben ser optimizados para mejorar la capacidad de medición del sistema.

Los resultados obtenidos con este montaje para la medición in situ con un sistema de espectrometría gamma fueron satisfactorios. Con los mismos se logra predecir la variación de la eficiencia del montaje en función del cambio de dos variables relevantes en el momento de medir descargas de ${ }^{131} \mathrm{I}$, como lo son la distancia entre la base de la caja de inspección y el detector, y la altura de la columna de líquido en la caja. Un desarrollo posterior de interés se relaciona con el estudio de un sistema el sistema de flujo dinámico como el que realmente se presenta en los sistemas de manejo de vertimientos de las instalaciones donde se realiza terapia con ${ }^{131} \mathrm{I}$.

Los límites de detección son función, entre otras variables, del tiempo de medición, teniendo en cuenta el orden de magnitud de la eficiencia de detección del sistema [15], y considerando tiempos de medición de 2 horas, la metodología tiene límites de detección del orden de $3 \mathrm{kBq} / \mathrm{l}$.

Teniendo en cuenta que en esta primera aproximación se consideró la distribución homogénea del radionúclido en el líquido, es necesario estudiar en un trabajo posterior el valor de incertidumbre introducida por este factor, debido al efecto de una posible no homogeneidad.

El montaje se construyó para determinar los parámetros de eficiencia, incertidumbre y límite de detección y con esto realizar mediciones en los puntos de descarga de las 
instalaciones generadoras (cajas de inspección) con una geometría estandarizada de área transversal $(60 \mathrm{~cm} \mathrm{x}$ $60 \mathrm{~cm}$ ), la metodología contiene algunos supuestos que son aplicables a las condiciones reales de medida. Sin embargo, para geometrías diferentes a la experimental o condiciones que se alejen de las aplicadas en el montaje es necesario estimar las correcciones que apliquen a la eficiencia de medición $\boldsymbol{\varepsilon}$.

Dadas las particularidades de cada sistema de detección que pueda emplearse, los valores de $\boldsymbol{\varepsilon}$ obtenidos en los resultados son específicos y aplicables únicamente para el modelo propuesto y el equipo de detección empleado en el experimento. Los límites de detección obtenidos se consideran compatibles con los niveles de actividad de descarga máximo de ${ }^{131} I$ establecidos en las recomendaciones [16].

\section{CONCLUSIONES}

El montaje propuesto contribuye de manera preliminar a dilucidar las diferentes variables que son necesarias para la puesta a punto e implementación de un protocolo para la determinación in situ por espectrometría gamma de alta resolución (GeHp) de descargas líquidas de ${ }^{131} \mathrm{I}$ en servicios de terapia. A partir del desarrollo se permite la evaluación rápida, sin contacto físico con los vertimientos radiactivos y con las especificaciones metrológicas aptas para medir este radionúclido en concentraciones comparables con los límites de vertimientos recomendados internacionalmente.

Aunque esta metodología puede ser aplicada a descargas pulsadas con concentración variables, ya que está basada en la integral de cuentas durante el tiempo de medición y no en valores instantáneos de las tasas de conteo, es necesario evaluar el efecto en el límite de detección de la misma para flujos grandes donde la concentración de actividad absoluta puede bajar a niveles no medibles.

\section{REFERENCIAS}

[1] E. O. Ruiz, "La Medicina Nuclear," Academia Nacional de Medicina - Asociación Colombiana de Medicina Nuclear, p. 13, 2002.
[2] United Nations Scientific Committee on the Effects of Atomic Radiation and UNSCEAR 2008. Report to the General Assembly with Scientific Annexes, "Sources and effects of Ionizing Radiation," 2010.

[3] International Commission on Radiological Protection, "Release of patients after therapy with unsealed radionuclides," Ann. ICRP, vol. 34, no. 2, pp. 1-79, 2004.

[4] International Atomic Energy Agency - IAEA, "Safety Reports Series No 63, Release of Patients After Radionuclide therapy," Vienna, 2009.

[5] International Atomic Energy Agency-IAEA, "Setting Authorized Limits for Radioactive Discharges: Practical Issues to Consider," Vienna, 2010.

[6] R. Barquero, F. Basurto, C. Núñez, and R. Esteban, "Liquid discharges from patients undergoing ${ }^{131} \mathrm{I}$ treatments," J. Environ. Radioact., vol. 99, no. 10, pp. 1530-1534. Oct. 2008.

[7] Ministerio de Minas y Energía, Resolución 41178 "Por la cual se modifica la Resolución 18 0005." Colombia, 2016.

[8] Ministerio de Minas y Energía, Resolución número 18 0005, Reglamento para la gestión de los desechos radiactivos. Colombia, 2010, pp. 1-84.

[9] E. Krawczyk, F. Piñero-García, and M. a FerroGarcía, "Discharges of nuclear medicine radioisotopes in Spanish hospitals," J. Environ. Radioact., vol. 116, pp. 93-8, Mar. 2013.

[10] P. S. Rose, R. L. Swanson, and J. K. Cochran, "Medically-derived ${ }^{131} \mathrm{I}$ in municipal sewage effluent," Water Res., vol. 46, no. 17, pp. 5663-5671, 2012.

[11] C. Tsabaris, D. L. Patiris, A. P. Karageorgis, G. Eleftheriou, V. P. Papadopoulos, D. Georgopoulos, E. Papathanassiou, and P. P. Povinec, "In situ radionuclide characterization of a submarine groundwater discharge site at Kalogria Bay, Stoupa, Greece," J. Environ. Radioact., vol. 108, pp. 50-59, 2012. 
[12] R. Berndt and P. Mortreau, "Performance test of the Multi-Channel Analyzer MCA-527 for Nuclear Safeguards Applications," Report EUR 26165 EN, 2013.

[13] M. Bé, V. Chisté, C. Dulieu, V. Chechev, N. Kuzmenko, and M. Galán, Table of Radionuclides (Vol. 1 - A = 1 to 150). Pavillon de Breteuil, 2004.

[14] Empresa de Acueducto y Alcantarillado de Bogotá -EAAB-, Requisitos mínimos para cajas de inspección externa para efluentes industriales. 2001, pp. 1-4.
[15] International Organization for Standardization - ISO, "ISO 11929:2010 - Determination of the characteristic limits (decision threshold, detection limit and limits of the confidence interval) for measurements of ionizing radiation -- Fundamentals and application," 2010.

[16] International Atomic Energy Agency - IAEA, "Clearance of materials resulting from the use of radionuclides in medicine, industry and research - TECDOC 1000," Vienna, 1998. 